\title{
Lattice Gauge Theory Simulations at Nonzero Chemical Potential in the Chiral Limit
}

\author{
I. M. Barbour and S. E. Morrison \\ Department of Physics and Astronomy, University of Glasgow, Scotland \\ (UKQCD collaboration) \\ John B. Kogut \\ Physics Department, University of Illinois at Urbana-Champaign, Urbana, IL 61801-30
}

(June 3, 2017)

\begin{abstract}
We present a method of simulating lattice QCD at nonzero chemical potential in the chiral limit. By adding a weak four-fermi interaction to the standard staggered fermion SU(3) QCD action, we produce an algorithm in which the limit of massless fermions is well-behaved and physical. Using configurations at zero chemical potential, and an exact fugacity expansion of the fermion determinant, we can simulate QCD at nonzero chemical potential and evade the notorious problem of the complex action. Small lattice simulations give physical results : At strong gauge coupling the critical chemical potential $\mu_{c}$ agrees with theoretical expectations and at weak gauge coupling $\mu_{c}$ is nonzero in the low temperature confined phase of QCD and jumps to zero in the high temperature quark-gluon plasma phase. In all these simulations the quarks are exactly massless and there is a Goldstone pion.
\end{abstract}

12.38.Mh, 12.38.Gc, 11.15.Ha

Typeset using REVTEX 


\section{INTRODUCTION}

One of the goals of Lattice Gauge Theory is a thorough investigation of the phase diagram of QCD in the temperature-chemical potential plane. Considerable progress has been made in simulating lattice QCD at nonzero temperature [1], but there has been little progress in understanding the theory at nonzero chemical potential $\mu$ even though a sound lattice formulation was presented twelve years ago [2]. One expects on the basis of elementary physics arguments [2] that as the chemical potential $\mu$ increases QCD will experience a transition to a quark-gluon plasma at one-third the mass of the proton, and that the chiral condensate will serve as an order parameter. The basic difficulty in simulating QCD at finite density and investigating the transition quantitatively is that the effective action resulting from the Grassmann integration over the fermions is complex due to the introduction of the chemical potential in the Dirac matrix. The standard simulation algorithms [3], for for lattice QCD with dynamical fermions do not apply to this situation. In order to avoid the determinant, early simulations used the quenched approximation, but they led to an unphysical value of the critical chemical potential $\mu_{c}$, namely, its proportionality to the pion mass [5]. The physical and mathematical reasons for this failure have been the subject of considerable debate [6]. Recent work by M. Stephanov [7], however, has shown that the quenched model is the $n_{f} \rightarrow 0$ limit of a theory with both quarks and conjugate quarks, and is not relevant to QCD with dynamical fermions.

Here we wish to advocate a new approach to simulating QCD with dynamical fermions at nonzero chemical potential which has two unique features. First, we approach the chiral limit of lattice QCD by adding a four-fermi term to its action but setting the bare masses of the quarks to zero. The four-fermi term is a perturbatively irrelevant interaction so it does not have an effect on the values of physical quantities in the theory's continuum limit. However, in the new algorithm, called $\chi$ QCD [8], the dynamical mass of the quark enters its propagator directly and makes the limit of massless quarks more accessible, as we will see below. The physics of massless Goldstone pions can be studied in $\chi \mathrm{QCD}$ without the need 
for extrapolation. In addition, the algorithm runs one to two orders of magnitude faster than the traditional one because a physical quantity, the dynamical quark mass rather than the bare lattice mass, controls the convergence of the inversion of the quark propagator, the most time consuming step in the procedure. $\chi \mathrm{QCD}$ approaches the chiral limit of QCD in a different direction than the standard action and appears to avoid some of its problems. Second, the fermion determinant is calculated exactly at any $\mu$ and any bare quark mass $m_{q}$, given a configuration produced by the $\chi \mathrm{QCD}$ algorithm at vanishing $\mu$ and any other $m_{q}$. This can be done because of the simple way $m_{q}$ and $\mu$ enter the theory's action. A large ensemble of configurations will generally be needed to produce observables at nonzero $\mu$ values with acceptable statistical errors. Preliminary simulations of the method have been successful and will be presented below.

\section{FORMULATION}

Consider QCD with a chiral 4-fermion interaction, ' $\chi$ QCD' [8]. The molecular dynamics Lagrangian for the lattice theory is

$$
\begin{aligned}
L= & -\beta \sum_{\square}\left[1-\frac{1}{3} \operatorname{Re}\left(\operatorname{Tr}_{\square} U U U U\right)\right]+\sum_{s} \dot{\psi}^{\dagger} M^{\dagger} M \dot{\psi} \\
& -\sum_{\tilde{s}} \frac{1}{8} N_{f} \gamma\left(\sigma^{2}+\pi^{2}\right)+\frac{1}{2} \sum_{\tilde{s}}\left(\dot{\sigma}^{2}+\dot{\pi}^{2}\right) \\
& +\frac{1}{2} \sum_{l}\left(\dot{\theta}_{7}^{2}+\dot{\theta}_{8}^{2}+\dot{\theta}_{1}^{*} \dot{\theta}_{1}+\dot{\theta}_{2}^{*} \dot{\theta}_{2}+\dot{\theta}_{3}^{*} \dot{\theta}_{3}\right)
\end{aligned}
$$

where

$$
M=\not D+m_{q}+\frac{1}{16} \sum_{i}\left(\sigma_{i}+i \epsilon \pi_{i}\right)
$$

and $\beta=6 / g^{2}, \gamma=1 / G^{2}, \epsilon=(-1)^{x+y+z+t}$, the $\theta_{i}$ parametrize the $\mathrm{SU}(3)$ link variables $U, \tilde{s}$ labels dual sites [9] on the lattice of volume $V=n_{t} \times n_{s}^{3}$, and we have introduced auxiliary fields $\sigma$ and $\pi$ to formally linearize the 4 -fermi term. We use staggered quarks in Eq. 1 because of their relatively good chiral symmetry properties. For simplicity we consider a 
theory where the 4-fermion operator has the

$$
U(1) \times U(1) \subset S U\left(N_{f}\right) \times S U\left(N_{f}\right)
$$

flavor symmetry generated by $\left(1, i \gamma_{5} \xi_{5}\right)$ [10]. This Lagrangian describes 8 flavors. For $N_{f}$ which is not a multiple of 8 we use "noisy" fermions [4] and multiply the fermion kinetic term by $N_{f} / 8$.

The Dirac operator of standard lattice QCD becomes singular as $m_{q} \rightarrow 0$. However, inspecting Eq. 2, we see that in $\chi$ QCD the Dirac operator remains non-singular at $m_{q}=0$ because the auxiliary field $\sigma$ develops a vacuum expectation value due to chiral symmetry breaking. Conjugate gradient inversion of the regular QCD Dirac operator requires a number of iterations which diverges as $V \rightarrow \infty$ and $m_{q} \rightarrow 0$. Inversion of the $\chi$ QCD Dirac operator requires a finite number of iterations even at $m_{q}=0$. In addition, the scale of the 'time' step in the molecular dynamics algorithm of $\chi \mathrm{QCD}$ is set by the dynamical quark mass and can be chosen several times larger here than in the ordinary lattice QCD algorithm, for the same systematic error [4] or acceptance rate [3].

In order to circumvent the difficulty of investigating the finite-density transition for dynamical fermions, we build on the method of reference [11]. The method was inspired by the classic work of Yang and Lee [12] who showed that the distribution of the zeros of a partition function determines the equation of state for a many-body system. For an Ising model in a magnetic field and for a lattice gas, it was shown that the zeros of the corresponding partition function lie on a unit circle in the complex fugacity plane. For a finite system these zeros will never lie in the physical range for the fugacity, namely the positive real axis. However, for a temperature below the phase transition, they will approach the real axis as the volume of the system tends to infinity. The singularities of the free energy in the thermodynamical limit are therefore obtained as the infinite-volume extrapolation of the zeros of the partition function. A detailed investigation of the finite-size scaling of the zeros enables one to evaluate the order of the phase transition and the critical exponent for the order parameter 13 . 
This proposed study of lattice QCD at finite density is based on expanding the Grand Canonical Partition Function $(\mathrm{GCPF})$ as a polynomial in the fugacity variable $\left(e^{\mu n_{\tau}}\right)$ where $n_{\tau}$ is the temporal extent of the lattice.

The GCPF is given as an ensemble average of the determinant of the Dirac operator normalised with respect to the determinant at $\mu=0$ :

$$
Z=\frac{\int[d U]\left[d U^{\dagger}\right] \operatorname{det} \hat{M}(\mu) \operatorname{det} M(\mu) e^{-S_{g}\left[U, U^{\dagger}\right]}}{\int[d U]\left[d U^{\dagger}\right] \operatorname{det} \hat{M}(\mu=0) \operatorname{det} M(\mu=0) e^{-S_{g}\left[U, U^{\dagger}\right]}}
$$

The Dirac fermion matrices $M$ and $\hat{M}$ are given by:

$$
\begin{gathered}
2 i M_{x y}(\mu)=Y_{x y}+G_{x y}+V_{x y} e^{\mu}+V_{x y}^{\dagger} e^{-\mu} \\
-2 i \hat{M}_{x y}(\mu)=Y_{x y}^{\dagger}+G_{x y}+V_{x y} e^{\mu}+V_{x y}^{\dagger} e^{-\mu} .
\end{gathered}
$$

where $Y=2 i\left(m_{q}+\frac{1}{16} \sum_{<x, \tilde{x}>}(\sigma(\tilde{x})+i \epsilon \pi(\tilde{x}))\right) \delta_{x y}$, G contains all the spacelike links and $\mathrm{V}$ all the forward timelike links.

The determinant of the Dirac operator is complex (for $\mu \neq 0$ ) in the $N_{c}=3$ case because $e^{\mu}$ favors forward propagation through Eq. 4 while $e^{-\mu}$ hinders backward propagation [2].

The determinants of the fermion matrices $M$ and $\hat{M}$ are related to that of the propagator matrix [14

$$
P=\left(\begin{array}{cc}
-G V-Y V & V \\
-V & 0
\end{array}\right)
$$

by $\operatorname{det}(2 i M)=e^{N_{c} \mu n_{s}^{3} n_{t}} \operatorname{det}\left(P-e^{-\mu}\right)$ and $\operatorname{det}(2 i \hat{M})=e^{N_{c} \mu n_{s}^{3} n_{t}} \operatorname{det}\left(\left(P^{-1}\right)^{\dagger}-e^{-\mu}\right)$ on an $n_{s}^{3} \times n_{t}$ lattice.

Since the eigenvalues of $P$ have a $Z\left(n_{t}\right)$ symmetry (since $P$ is proportional to $V$ ) and because the complex conjugate configuration is equally probable in the ensemble average, the GCPF can be expanded as

$$
Z=\sum_{n=-2 N_{c} n_{s}{ }^{3}}^{2 N_{c} n_{s}{ }^{3}}<b_{|n|}>e^{n \mu n_{t}}=\sum_{n=-2 N_{c} n_{s}{ }^{3}}^{2 N_{c} n_{s}{ }^{3}} e^{-\left(\epsilon_{n}-n \mu\right) / T} .
$$

The $b_{n}$ are determined from the eigenvalues of $P^{n_{t}}$. 
For $N_{c}=3$, the tunneling between the different $Z(3)$ vacua should eliminate the triality non-zero coefficients. In the simulations described below we do observe strong cancellations in these coefficients for $n=0$ around zero.

The zeros of this polynomial are the Lee-Yang zeros in the complex fugacity plane. Their distribution and behaviour with respect to finite volume scaling will be discussed in a future publication.

\section{RESULTS}

We consider a description of the system in terms of the canonical partition functions for fixed particle number [15]. The chemical potential as a function of the baryon number density is obtained from the local derivative of the energy, $\epsilon_{n}$, of the state with $n$ fermions with respect to $n$.

$$
\mu(\rho)=\frac{1}{2 N_{c} n_{s}^{3}} \frac{\partial \epsilon_{n}}{\partial \rho}
$$

where $\rho$ is the fermion density, $\frac{n}{2 N_{c} n_{s}^{3}}$.

Although some of the $\epsilon_{n}$ are determined with large error and have imaginary part $i \pi$ because the corresponding $b_{n}$ is negative (in principle, many more measurements should give all triality zero $b_{n}$ positive), it is clear from the simulations that, for all $\bmod (\mathrm{n}, 3)=0$, their real parts form a continuous curve in $n$, as shown in Fig. 1.

With this assumption we made a cubic spline fit to a randomly selected subset of $n_{s}^{3} / 3$ of the $2 n_{s}^{3} \epsilon_{n}$ 's with triality zero and evaluated the derivative. This process was performed $n_{s}^{3} / 3$ times and an estimate of the fitting error and the mean estimated from the distribution of the corresponding $\mu(\rho)$ resulted. 


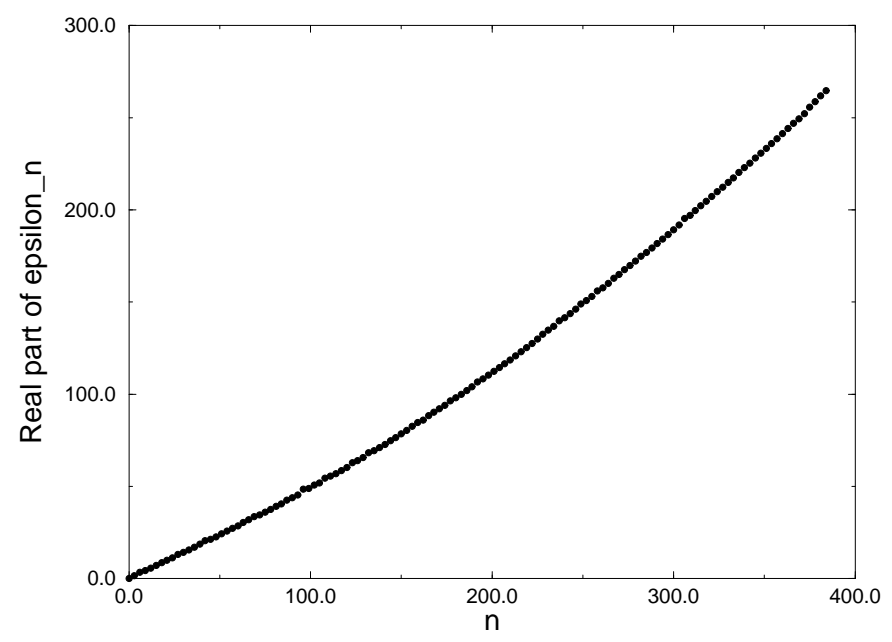

FIG. $1 . \epsilon_{n}$ vs. $n, 6^{4}$ lattice, $\beta=5.0$ and $\gamma=5.0$

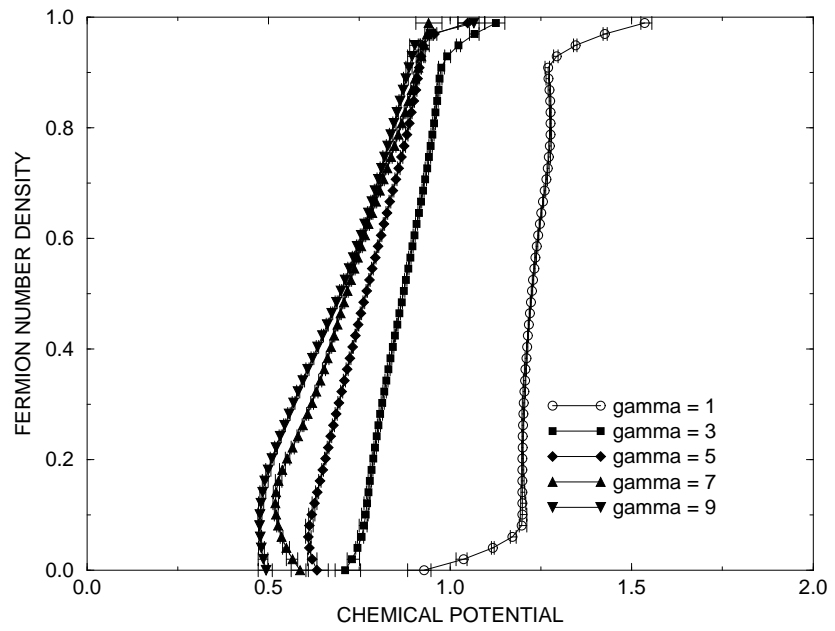

FIG. 2. Fermion density vs. chemical potential, $4^{4}$ lattice, $\beta=0.5$ 


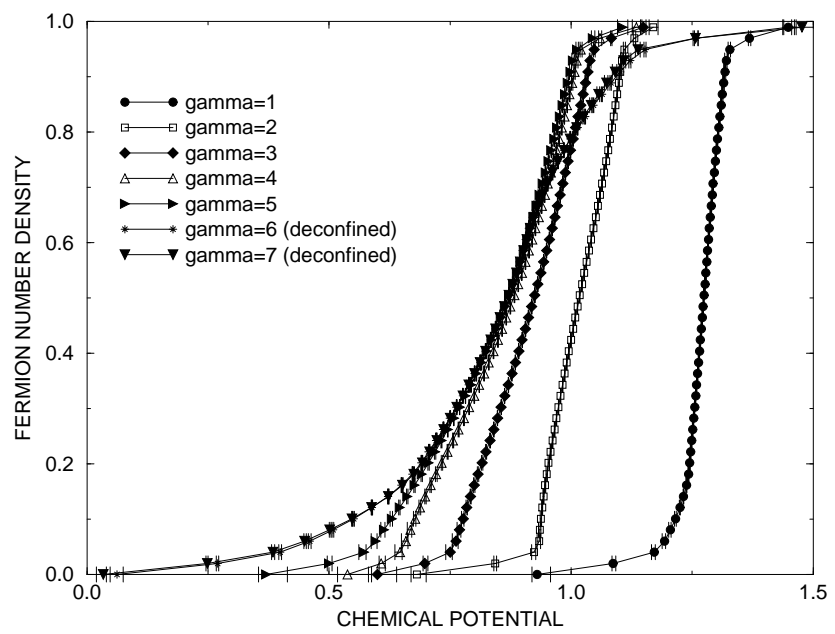

FIG. 3. Fermion density vs. chemical potential, $6^{4}$ lattice, $\beta=5.0$

Our preliminary simulation results from small lattices are very encouraging. In Fig. 2 we show strong gauge coupling $\left(\beta=0.5, m_{q}=0\right)$ results of the induced fermion number vs. chemical potential $\mu$ on a $4^{4}$ lattice at various four-fermi couplings $\gamma$. Since the pion is strictly massless in these simulations and since $\mu_{c}$ is distinctly nonzero for each parameter choice, the simulation is not afflicted by the diseases of the quenched standard action. In fact, for large $\gamma$ where the action reduces to the usual lattice QCD action, mean field analyses [16], which should be reliable at strong gauge coupling, predict $\mu_{c}$ near 0.60 , in rough agreement with our figure.

Next we simulated a weaker gauge coupling in order to approach the continuum limit of the theory and test the simulation method more strenuously. We chose $\beta=5.0, m_{q}=0$ for various $\gamma$ on a larger $6^{4}$ lattice. Simulations at zero $\mu$ were performed and measurements of the chiral condensate and heavy quark potential indicated a finite 'temperature' quark gluon plasma transition between $\gamma$ of 5.0 and 6.0. (At $\gamma=5.0$ we measured $<\sigma\rangle=.20$ and the Wilson Line $\langle W L\rangle=.01$, while at $\gamma=6.0$ we found $\langle\sigma\rangle=.03$ and $\langle W L\rangle=.28$.) The task for our new algorithm was to find this transition and measure the critical chemical potentials in the 'cold' hadronic phase for $\gamma$ below 5.0 and do the same in the 'hot' plasma phase for $\gamma$ above 6.0. Our simulation results, shown in Fig. 3, show this transition very 
clearly - for $\gamma$ of 5.0 and smaller the $\mu_{c}$ is above 0.50 , while at $\gamma=6.0 \mu_{c}$ falls abruptly to zero, indicating the presence of unconfined, 'massless' quarks. Clearly, this estimate of $\mu_{c}$ is crude. Simulations of simpler models on much larger lattices [17] suggests that the 'tails' in the curves in Fig. 3 are finite size effects. The finite temperature transition of $\chi$ QCD is being studied on larger lattices for $\gamma \geq 10$ at variable $\beta$ and more observables are being measured [8] to obtain quantitative predictions for pure QCD.

Preliminary measurements, on $4^{4}$ and $6^{4}$ lattices, of the fermion energy density and condensate using the stochastic estimator method and including the ratio of the determinants of the Dirac operator at $\mu$ to that at $\mu=0$ are consistent with the behaviour of the number density found by the above method.

At the present time extension to larger lattices is limited by the algorithm used to determine the eigenvalues of the propagator matrix. The cpu time and storage, to a first approximation, scale as $n_{s}^{6}$ but are independent of $n_{t}$. The algorithm has been successfully implemented on an $8^{4}$ lattice and should be viable on a $10^{3} \times n_{t}$ lattice at its present stage of development.

\section{CONCLUSIONS}

Clearly much more work along these lines is called for - much larger lattices must be used to extract continuum physics. Although in principle the four fermi term is irrelevant and the value of $\gamma$ should not effect the values of observables in physical units in the continuum limit, practical simulations on finite lattices should be done with the four fermi coupling sufficiently small. This issue is discussed briefly in [8], but more quantitative work is needed and is underway. Nonetheless, the method has passed several nontrivial tests which we find encouraging.

We are also hopeful that $\chi \mathrm{QCD}$ can be used to improve the action of lattice QCD, speed up spectroscopy and matrix element calculations of conventional hadronic phenomenology, and determine the universality class of the finite temperature hadronic matter/quark gluon 
plasma transition [18] [19]. An algorithm which runs directly in the chiral limit will be very helpful here.

\section{ACKNOWLEDGEMENTS}

This work was partially supported by NSF under grant NSF-PHY92-00148, by PPARC under grant GR/K55554 and by NATO under grant CRG960002. The simulations were done on the CRAY C-90's at PSC and NERSC. The authors thank Ely Klepfish, D.K. Sinclair, M. Stephanov, M.-P. Lombardo and A. Kocić for discussions. 


\section{REFERENCES}

[1] K. Kanaya, Nucl. Phys. B47, 47 (1996).

[2] J.B. Kogut, H.Matsuoka, M. Stone, H.W. Wyld, S. Shenker, J. Shigemitsu and D.K. Sinclair, Nucl. Phys. B225 [FS], 93 (1983). P. Hasenfratz and F. Karsch, Phys. Lett. B125, 308 (1983).

[3] S. Duane, A.D. Kennedy, B.J. Pendleton and D. Roweth, Phys. Lett. B195, 216 (1987).

[4] S. Duane and J.B. Kogut, Phys. Rev. Lett. 55, 2774 (1985). S. Gottlieb, W. Liu, D. Toussaint, R.L. Renken and R.L. Sugar, Phys. Rev. D35,2531 (1987).

[5] C.T.H. Davies and E.G. Klepfish, Phys. Lett. B256, 68 (1991).

[6] J.B. Kogut, M.-P. Lombardo and D.K. Sinclair. Phys. Rev. D51, 1282 (1995); Phys. Rev. D54, 2303 (1996).

[7] M. Stephanov, Phys. Rev. Lett. 76, 4472(1996).

[8] J.B. Kogut and D.K. Sinclair, hep-lat/9607083.

[9] Y. Cohen, S. Elitzur and E. Rabinovici, Nucl. Phys. B220, 102 (1983).

[10] S. Hands, A. Kocić and J.B. Kogut, Ann. of Phys. 224, 29 (1993) 29.

[11] I.M. Barbour and A.J. Bell, Nucl. Phys. B372, 385 (1992).

[12] C.N. Yang and T.D. Lee, Phys. Rev. 87, 404 (1952). T.D. Lee and C.N. Yang, ibid 410.

[13] C. Itzykson, R.B. Pearson and J.B. Zuber, Nucl. Phys. B220[FS8], 415 (1983).

[14] P. Gibbs, Phys. Lett. B182, 369 (1986)

[15] For example, see R.P. Feynman, Statistical Mechanics (W.A. Benjamin,1972).

[16] N. Bilić, K. Demeterfi and B. Petersson, Nucl. Phys. B377, 651 (1992).

[17] S. Hands, S. Kim, and J.B. Kogut, Nucl. Phys. B442, 364 (1995). 
[18] R. Pisarski and F. Wilczek, Phys. Rev. D29, 338 (1984).

[19] A. Kocić and J. B. Kogut, Phys. Rev. Lett. 74, 3109 (1995). 